\title{
ESTRATÉGIAS PARA A IMPLANTAÇÃO DE UMA NOVA PROPOSTA PEDAGÓGICA NA ESCOLA DE ENFERMAGEM DA UNIVERSIDADE FEDERALDA BAHIA
}

\author{
Josicelia Dumêt Fernandes* \\ Silvia Lucia Ferreira** \\ Maristela P. S. La Torre*** \\ Darci De Oliveira Santa Rosa**** \\ Heloniza O. G. Costa*****
}

\section{Resumo}

Este artigo apresenta uma reflexão sobre o processo de construção do Projeto Pedagógico da Escola de Enfermagem da UFBA. A socialização dessa experiência pretende contribuir com outras instituições de ensino de enfermagem que, para atender às novas DCENF, passarão a vivenciar processos semelhantes ou que, no momento, passam pelo mesmo processo. O artigo traz alguns antecedentes e a fundamentação legal da mudança, apresentando os pressupostos que norteiam a atual proposta, bem como a organização curricular, os principais desafios e algumas estratégias de superação dos mesmos. Embora sejam apontados alguns aspectos que precisam de aprofundamento por parte do corpo docente, como a transversalidade de conteúdos, a gestão acadêmica, o processo de avaliação e a operacionalização da proposta, as autoras concluem que o debate não se esgota neste momento. Por se tratar de um processo dinâmico em construção, outros desafios poderão surgir exigindo novas estratégias para seu (re) direcionamento.

Descritores: Diretrizes Curriculares; Enfermagem; Projeto Pedagógico

\section{Abstract}

This article reflects on the process of designing the Pedagogical Project for the Nursing School from Federal University of Bahia. The socialization of this experience intends to contribute with other nursing institutions which, in order to meet new curricular requirements (DCENF - Nationnal Curricular Guidelines for Nursing, Medicine, and Nutrition Undergraduate Programs), are going to experience similar processes or which are presently going through the same process. This article includes some historical features and the legal basis for changes, by presenting the reasons for the current proposal, as well as its curricular organization, the main challenges and some strategies for overcoming them. Although some aspects which still need further attention from the faculty are presented, such as interdisciplinary program contents, academic management, the evaluation process and the implementation of the proposal, the authors come to the conclusion that this debate is to be continued. As this process is a dynamic one and is still under construction, other challenges might come up that demand the use of new strategies for their (re)orientation.

Descriptos: curricular guidelines; nursing; pedagogical project

Title: Strategies for the implementation of a new pedagogical proposal at the Nursing School from Federal University of Bahia
\end{abstract}

\section{Resumen}

El artículo presenta una reflexión sobre el proceso de construcción del Proyecto Pedagógica de la Escuela de Enfermería de la UFBA. La socialización de la experiencia pretende contribuir con otras instituciones de enseñanza de enfermería, pues para atender a las nuevas Directrices, pasarán a vivenciar procesos semejantes. El artículo trae algunos antecedentes y los fundamentos legales del cambio y explica los presupuestos que orientan la actual propuesta, la organización curricular, los principales desafíos y algunas estrategias para superarlos. Aunque se apunten algunos aspectos que el cuerpo docente tendrá que profundizar, tales como la transversalidad de contenidos, la gestión académica, el proceso de evaluación y el cómo llevar a cabo la propuesta, las autoras concluyen que el debate no se agota, pues se trata de un proceso dinámico en construcción y otros desafíos podrán surgir, exigiendo, por lo tanto, nuevas estrategias.

Descriptores: directrices curriculares; enfermería; proyecto pedagógico

Título: Estrategias para implementar una nueva propuesta pedagógica en la Escuela de Enfermería de la Universidad Federal de Bahia

\section{Antecedentes da mudança}

Antes mesmo da aprovação das Diretrizes Curriculares Nacionais para o Curso de Graduação em Enfermagem, desencadeada pelo Edital 04/97 do MEC/SESU, a Escola de Enfermagem da Universidade Federal da Bahia (EEUFBa) reestruturou sua comissão de acompanhamento e avaliação do currículo existente, com objetivo de acompanhar e avaliar o currículo implantado nos moldes da Portaria 1.721/94 do MEC, no sentido de direcionar o curso para o atendimento das necessidades atuais da formação. O currículo tradicional já não dava conta dos problemas complexos colocados pela sociedade e estava provocando insatisfação entre os docentes e os estudantes, pois eram muitas as distorções decorrentes da fragmentação curricular, da dicotomia teoria/prática, curativo/ preventivo, individual/coletivo, ciclo básico/ciclo profissionalizante e tantas outras distorções desse modo de organização do currículo.
A EEUFBa, à semelhança de outras escolas/cursos, vinha desenvolvendo suas ações pedagógicas através do ensino centrado nas(os) professoras(es), na transmissão de conteúdos, na avaliação somativa, com enfoque na área cognitiva (memorização apenas de conteúdos teóricos muitas vezes descontextualizados), deixando as áreas afetivas e motoras, aparentemente, em segundo plano.

Frente a essa realidade, a construção de um projeto pedagógico inovador se constitui numa árdua tarefa institucional, não só pela dificuldade que traz a construção do novo, mas, sobretudo, pelo enfrentamento dos distintos entendimentos e interesses que mudanças dessa natureza provocam - por um lado, existem grupos que acham desnecessárias as mudanças, pela segurança que os princípios e práticas tradicionalmente organizados dão, por outro lado, as incertezas do como inovar, como reconstruir, como fazer diferente e romper com o estabelecido no processo de formação da(o) enfermeira(o). O

* Professora Titular da Escola de Enfermagem da UFBa. Doutora em Enfermagem.

** Professora Adjunta da Escola de Enfermagem da UFBa. Pós Doutora em Enfermagem

*** Professora Adjunta da Escola de Enfermagem da UFBa. Mestra em Enfermagem.

**** Professora Adjunta da Escola de Enfermagem da UFBa. Doutora em Enfermagem.

*****Professora Assistente IV da Escola de Enfermagem da UFBa. Mestra em Saúde Comunitária e Doutoranda em Administração.

E-mail do autor: silvialf@ufba.br 
reordenamento dessa lógica, muito estabelecida, para uma lógica pedagógica que considere estudantes como sujeitos do seu processo de formação, que assegure a formação de profissionais críticos, criativos e comprometidos com a transformação dessa sociedade desigual e excludente, implica no enfrentamento da complexidade trazida pela realidade da prática profissional.

A iniciativa de mudança dessa realidade fundamentouse no entendimento da necessidade de formar profissionais aptos a aprender a aprender e comprometidos com o enfrentamento dos graves problemas da nossa sociedade. A fundamentação teórica desse entendimento insere-se nas premissas da educação contemporânea: aprender a conhecer, aprender a fazer, aprender a viver juntos e aprender a ser ${ }^{(1)}$.

\section{A base legal}

A LDB - Lei 9.394 de 20 de dezembro de 1996(3), que estabelece as diretrizes e bases da educação nacional, traz no seu bojo uma alteração significativa nas bases da educação brasileira, abrindo espaços para a flexibilização dos currículos de graduação do país e favorecendo a superação do modelo de currículo mínimo e da grade curricular com um número excessivo de disciplinas interligadas por um sistema rigoroso de pré-requisitos. Essa flexibilização descortina possibilidades às instituições de ensino superior para implementarem Projetos Pedagógicos inovadores, numa perspectiva de mudança para a formação profissional e, finalmente, a adoção de Diretrizes Curriculares para cada curso.

O processo de formação na área da saúde conta com o Parecer do CNE/CES No 1133 de 7/8/2001(4) que apresenta os documentos referenciais, assim como a concepção de saúde, os princípios e diretrizes do Sistema Único de Saúde (SUS) e o objeto e objetivo das Diretrizes Curriculares dos Cursos de Graduação na área.

A Resolução CNE/CES No 3 de 7/11/2001(2) aprova as Diretrizes Curriculares Nacionais dos Cursos de Graduação em Enfermagem (DCEnf) que se constituem num instrumento norteador do processo de construção de Projetos Pedagógicos, trazendo como parâmetros: um eixo norteador dos conteúdos mínimos para a formação do profissional; flexibilidade; formação integral; incorporação de atividades complementares em relação ao eixo fundamental do processo de formação; interdisciplinaridade; predominância da formação sobre a informação; articulação entre teoria e prática; indissociabilidade entre ensino, pesquisa e extensão(5)

Vale destacar, contudo, que apesar da sua função de instrumento norteador do processo de construção de Projetos Pedagógicos, as DCEnf não podem ser reduzidas a atos normativos, regras ou padrões lineares a serem, assepticamente, seguidos, nem, tampouco, a uma simples proposta de reordenamento de disciplinas e cargas horárias.

Os Projetos Pedagógicos, com base nas Diretrizes Curriculares Nacionais dos Cursos de Graduação em Enfermagem, devem estar fundamentados em princípios educacionais que garantam a flexibilidade dos currículos, a consideração dos estudantes como sujeitos do processo ensino-aprendizagem e dos professores como facilitadores desse processo, a articulação teoria/prática, a pesquisa integrada ao ensino e à extensão, metodologias ativas para o processo ensino/aprendizagem, diversificação dos cenários de aprendizagem, currículos fundamentados no humanismo, avaliação formativa, educação orientada aos problemas relevantes da sociedade e terminalidade dos $\operatorname{cursos}^{(5)}$.

Nessa perspectiva, este artigo, busca levantar algumas questões para subsidiar a implementação de mudanças na educação em enfermagem, trazendo a experiência da Escola de Enfermagem da Universidade Federal da Bahia (EEUFBa) na construção de um Projeto Pedagógico criativo e inovador que procura assegurar uma formação adequada às rápidas mudanças do mundo moderno. Espera-se, destarte, estar contribuindo com o movimento de transformação desenvolvido nas Escolas/Cursos de Enfermagem no que se refere à formação de enfermeiros como produtores de conhecimento e como sujeitos na construção de um novo modelo de atenção à saúde que atenda às reais necessidades de saúde do conjunto da população e que esteja voltado para o Sistema único de Saúde e sua consolidação.

\section{Os pressupostos}

Para o enfrentamento do desafio inicial foram desenvolvidas amplas discussões, mobilizando docentes, discentes e profissionais de serviço, buscando-se ampliar os espaços de diálogo, interlocução e ação conjunta com os sujeitos coletivos para a construção de uma prática inovadora. Essas discussões pautaram-se no entendimento de que quaisquer que fossem as medidas a serem adotadas, elas teriam que permitir a saída do pólo de ensino, centrada no professor, para o pólo da aprendizagem, centrado no aluno; a saída da fragmentação do modelo disciplinar para o currículo integrado; a saída da teoria que antecede a prática para a articulação teoria/prática; a saída das concepções de saúde como ausência de doença para a saúde enquanto condições de vida; a saída da polarização individual/coletivo e biológico/ social para uma consideração de interpenetração e transversalidade ${ }^{(6)}$.

Alcançando-se esse pensamento consensual, partiuse para a definição dos pressupostos teóricos, filosóficos e metodológicos que pudessem estar direcionando a construção do Projeto Pedagógico voltado para os princípios do SUS, da Ética, da Cidadania, do Processo Saúde/Doença e do Cuidado. Nesse sentido, a construção do Projeto Pedagógico da EEUFBa fundamentou-se nos seguintes pressupostos ${ }^{(7)}$ :

Aluno como sujeito - $\mathrm{O}$ aluno deve ser capaz de aprender a aprender, de reconhecer a transitoriedade do conhecimento científico, de identificar as lacunas do seu conhecimento, de saber buscar ativamente informações para resolução de problemas e de reconhecer e respeitar os saberes que orientam as ações dos demais profissionais e dos usuários.

Articulação teoria/prática - As ações pedagógicas devem conformar o equilíbrio teoria/prática para a construção das competências estimulando docentes e discentes a buscar novos conhecimentos em resposta às questões colocadas pela prática. Essas ações apontam para o princípio de que o aprender começa do fazer, para poder saber fazer e ter a capacidade de refazer.

Diversificação dos cenários de aprendizagem - O espaço da formação deve estar intrinsecamente ligado à realidade concreta numa contínua aproximação do mundo do ensino com o mundo do trabalho. Os vários campos do exercício profissional devem ser incluídos como espaços do processo ensino-aprendizagem, através da incorporação de estudantes e professores no processo de produção dos serviços, numa articulação efetiva que não se reduz ao uso desses espaços apenas como laboratórios para a aprendizagem. A participação de docentes, estudantes, agentes das práticas sanitárias e da comunidade deve ter a perspectiva de uma atuação conjunta que contribua tanto para a formação, como para imprimir mudanças na produção de serviços.

Pesquisa integrada ao ensino - A pesquisa deve estar incorporada como atitude cotidiana para o desenvolvimento da capacidade de estabelecer o questionamento reconstrutivo da vida e desenvolver a habilidade de produzir conhecimento próprio, assegurando uma assistência de qualidade.

Fundamentação no humanismo - O desenvolvimento de valores e atitudes no processo ensino/aprendizagem deve 
ser uma constante, uma vez que a prestação do cuidado é realizada por sujeitos portadores de valores, cultura, ideologias que permeiam a prática sanitária desses agentes. Além disso, é preciso levar em conta que todos os espaços de cuidado/atenção têm o potencial de serem instituintes de cidadania.

Metodologias ativas para o processo ensino-aprendizagem - Metodologias que possibilitem o aprender a aprender, que garantam o aprender fazendo e instaurem relações democráticas dentro das instituições de ensino e prestadoras de serviços; metodologias centradas nos estudantes, vistos como sujeitos do processo ensinoaprendizagem e como cidadãos; metodologias fundamentadas nos princípios da pedagogia interativa, na concepção pedagógica crítica e reflexiva, tendo como eixo central a participação ativa dos estudantes em todo o processo, incluindo todos os novos e diferentes cenários de prática.

Avaliação formativa - A avaliação deve estar voltada para as competências, traduzidas no desempenho, deixando de ser pontual, punitiva e discriminatória, orientada à esfera da cognição e memorização, para transformar-se num instrumento de acompanhamento de todo o processo ensino-aprendizagem, como forma de garantir o desenvolvimento das competências necessárias à formação do profissional.

Educação orientada aos problemas relevantes da sociedade - A realidade concreta e os reais problemas da sociedade devem ser tomados como substrato essencial para o processo ensino/aprendizagem, como forma de possibilitar a compreensão dos múltiplos determinantes das condições e vida e saúde da população.

Flexibilidade curricular - Adoção de medidas que contraponham a rigidez dos pré-requisitos e dos conteúdos obrigatórios ordenados em seqüência obrigatória, como se existisse apenas uma maneira de aprender.

Terminalidade do curso - Garantia da formação generalista, instrumentalizando o profissional a atuar nos mais variados contextos, opondo-se à especialização precoce e evitando visões parciais da realidade.

\section{A organização do curso}

Ao pensar a organização do curso, buscou-se novas formas de preparar profissionais enfermeiras(os). Essa nova forma implica na criatividade e compreensão como ferramentas fundamentais para o agir, assim como no compromisso e ética como elementos presentes em todos os momentos da formação e atuação profissionais. Assim, a opção colocada para dar conta dos princípios pedagógicos e do perfil esperado para $\mathrm{a}(0)$ enfermeira(o) foi a de romper com a rigidez das disciplinas, dos pré-requisitos e dos conteúdos obrigatórios (na maioria das vezes desconectados e distantes dos problemas e necessidades colocados pela realidade social), para a construção de um currículo integrado, onde o eixo da formação passa a ser a prática/trabalho/cuidado de enfermagem voltado para o processo da vida e da saúde dos indivíduos.

A proposta de organização do currículo integrado toma como eixo unidades temáticas, denominadas laboratórios, onde a prática/cuidado de enfermagem e a compreensão/ intervenção na realidade são assumidas como eixos articuladores do ensino, desde o início da vida acadêmica. Assume-se a transversalidade dos conteúdos trabalhados pelos campos de conhecimento disciplinar, articulando-se os conteúdos teórico-práticos, em função das demandas trazidas pelos problemas concretos da realidade e das competências a serem desenvolvidas, numa seqüência orientada pelo grau de autonomia no processo de cuidar. Esses laboratórios, por sua vez, buscam manter, na sua estruturação e operacionalização, coerência com os princípios pedagógicos assumidos.

Nessa perspectiva foram organizados seis Laboratórios a serem distribuídos em oito semestres, sendo, nos dois últimos, o Estágio Curricular com 810 horas, incluindo rede básica e rede hospitalar. A dinâmica organizacional desses Laboratórios será submetida a um processo contínuo de construção e reconstrução e permeado por uma efetiva interação entre o ensino e a prática profissional, pela integração entre prática e teoria, pela busca de soluções específicas e originais para diferentes situações, pela integração ensino-trabalhocomunidade e professor-aluno ${ }^{8}$.

Os conteúdos que compõem os Laboratórios estão postos de forma progressiva em nível de complexidade, partindo do autoconhecimento para o conhecimento do outro, seja ele sadio ou doente, vivendo em ambiente físico, social, político e psicológico adversos. Estão estabelecidos, também, os conteúdos considerados transversais, que são aqueles referentes às ciências humanas e sociais, assim como conteúdos comuns às áreas de atuação considerados importantes na formação da (0) enfermeira (0).

\section{Os principais desafios}

Ao se buscar a superação da fragmentação de um currículo por disciplinas para um currículo integrado, foi necessário superar a formação estanque de docentes, romper barreiras conceituais e compreender a relação de suas especificidades com as demais áreas do saber.

Romper com a divisão ciclo básico e ciclo profissionalizante não se constitui apenas num desafio ao interior da escola, mas, perpassa os muros da instituição e se coloca como um grande desafio que vai de encontro à organização da Universidade, refletindo-se nos planejamentos dos departamentos, apesar da grande motivação por parte de docentes que já estão mais familiarizados com a proposta.

A articulação teoria/prática também ultrapassa os muros da escola, através de um processo que possibilita às(aos) aluna(os), docentes e enfermeiras(os) de serviço, o aprender na prática com as experiências que começam pelo fazer, passam pelo saber fazer, para, finalmente, refazer. Esse processo coloca o desafio de teorizar a partir da prática, sem se restringir ao hospital ou laboratórios, mas incorporando os vários locais onde a vida acontece (família, comunidade, creches, escolas, local de trabalho entre outros). Espaços, estes, que se constituem numa fonte rica de aprendizagem. Para isso será necessário o estabelecimento de parcerias e/ou convênios com vistas à articulação e integração docente assistencial, capaz de dar conta da complexidade do desenvolvimento de projetos pedagógicos voltados para as reais condições de vida e saúde da população.

Além destes desafios que são decorrentes da mudança de princípios norteadores, outras dificuldades estão relacionadas às questões de infra-estrutura acadêmica e administrativa da instituição, conseqüentes do sucateamento das instituições públicas de ensino superior.

\section{Algumas estratégias na implementação da proposta}

Para superação das dificuldades, estão sendo estabelecidas estratégias que possam possibilitar a implantação do novo Projeto Pedagógico da EEUFBa, onde se destacam:

Estabelecimento de um programa de educação continuada para os docentes em Metodologia do Ensino Superior e Tutoria.

Criação de um espaço de encontros semanais de docentes para discussão de estratégias de avaliação e de técnicas pedagógicas de ensino e aprendizagem.

Criação de um banco de dados para orientação e uso dos docentes em formação pedagógica. 
- Definição de encontros interdisciplinares para discussão de situações que envolvem o cotidiano, despertando o interesse no aluno em analisar as dimensões da prática profissional na atenção à saúde individual e coletiva. Envolvendo docentes e discentes

- Criação de espaços para discussões e reflexões de conteúdos transversais e interdisciplinares para compreensão das questões ligadas à vida, à saúde, à cidadania, à ética, ao cuidado de enfermagem e à consolidação do SUS.

\section{Considerações finais}

Esse relato de experiência, evidentemente, não se completa por si só. Ele faz parte de um processo maior de implementação da mudança na formação do enfermeiro em nosso país. Ele é apenas o início de uma proposta inovadora que, enfrentando muitos desafios, está sendo encaminhada para implantação na Escola de Enfermagem da UFBA, ainda no ano de 2003.

É fundamental que todos os envolvidos nesse processo de mudança incorporem a filosofia das Diretrizes Curriculares Nacionais para o Curso de Enfermagem, repensando o contexto de saúde local, as práticas que hoje são implementadas e que queremos para o amanhã, na perspectiva de dar concretude aos princípios orientadores do SUS.

A construção de um Projeto Pedagógico para a formação da(o) enfermeira(o) é muito mais do que conseguimos apresentar, necessitando, destarte, de mais estudos em busca da sua implementação que perpassa o processo de trabalho em saúde.

\section{Referências}

1. Gadotti M. Pedagogia da práxis. São Paulo: Cortez; 1998.

2. Conselho Nacional e Educação (BR). Resolução CNE/CES n $n^{0} 3$ de 7 de novembro de 2001. Brasília (DF); 2001.

3. Ministério da Educação (BR). Lei n. ${ }^{\circ} 9.394$, de 20 de dezembro de 1996: estabelece as diretrizes e bases da educação nacional. Diário Oficial da União, Brasília (DF) 1996 dez 23;34(248)Seção 1:27.83341.

4. Conselho Nacional de Educação(BR). Parecer do CNE/CES no 1133 de 7 de agosto de 2001. Brasília (DF); 2001.

5. Xavier I, Fernandes JD, Ceribelli MI. Diretrizes curriculares: articulação do texto e contexto. Boletim Informativo da Associação Brasileira de Enfermagem, Brasília (DF) 2002 jul;44(2): 6-7.

6. Costa HOG. Diretrizes curriculares: a experiência da Escola de Enfermagem da UFBA. Boletim Informativo da Associação Brasileira de Enfermagem, Brasília (DF) 2002 jul;44(2):7.

7. Costa HOG, Araújo MJS, Santos MP, Azevedo NDA. Novas diretrizes curriculares para o ensino de enfermagem. In: Arruda BKG, organizador. A educação profissional em saúde e a realidade social. Recife (PE): IMIP; 2001. p. 279-290.

8. Ministério da Saúde (BR). Capacitação pedagógica para instrutor/ supervisor: área da saúde. Brasília (DF); 1989.

9. Universidade Federal da Bahia. Do péssimismo da razão para o otimismo da vontade: referências para a construção dos projetos pedagógicos nas IES brasileiras. O currículo como expressão do projeto pedagógico: um processo flexível. Salvador (BA): EDUFBA; 2001.

Data de recebimento: $30 / 08 / 2003$

Data de aprovação: 30/10/2003 